\title{
Can patients with interstitial lung disease safely undergo pulmonary resection?
}

\author{
Chukwumere Nwogu, MD, PhD
}

See related article on pages 64-70.

Interstitial lung disease (ILD) is a well-known risk factor for pulmonary complications after lung resections and presents a significant challenge for thoracic surgeons during preoperative risk assessment. A firm understanding of the incidence and consequences of ILD acute exacerbation after various types of pulmonary resection is necessary to accurately estimate postoperative morbidity and mortality. The use of predicted postoperative forced expiratory volume in 1 second to assess surgical risk is more applicable to patients with obstructive pulmonary disease. However, the use of forced vital capacity (FVC) and diffusion capacity of the lung for carbon monoxide (DLCO) in a similar manner, may be very useful for the selection of patients with ILD who may safely undergo pulmonary resection.

Various investigators have reported results after pulmonary resection in patients with ILD. Voltolini and colleagues ${ }^{1}$ from Italy identified a small cohort of 37 patients with ILD ( $4.8 \%$ of 775 lung resections) who had undergone lung resection over a 10 -year period. The patients with ILD had a higher incidence of postoperative acute respiratory distress syndrome (ARDS; $13.5 \%$ vs $2.3 \% ; P<.01$ ) and higher postoperative mortality ( $8 \%$ vs $1.4 \%, P<.01)$. In the ILD group, lower preoperative FVC (mean, $77 \%$ vs $93 \% ; P<.01)$ and lower Dlco $(47 \%$ vs $62 \% ; P<.01)$ were significantly associated with post operative ARDS. The analysis of the outcome related to specific surgical procedures also showed a significantly higher postoperative mortality for pneumonectomy $(25 \%$ vs $3.3 \% ; P<.01)$ and lobectomy $(6.6 \%$ vs $1.3 \% ; P<.01)$ in patients with ILD compared with patients without ILD. No patient died after a sublobar resection in the ILD group. Patients with ILD with a preoperative FVC less than $80 \%$ of predicted had $50 \%$ incidence of postoperative acute lung injury/ ARDS, whereas those with FVC greater than $90 \%$ of predicted had no incidence.

\footnotetext{
From the Roswell Park Cancer Institute, Buffalo, NY.

Disclosures: Author has nothing to disclose with regard to commercial support. Received for publication Sept 29, 2014; accepted for publication Sept 29, 2014 Address for reprints: Chukwumere Nwogu, MD, PhD, Thoracic Surgery Department, Roswell Park Cancer Institute, Elm \& Carlton Streets, Buffalo, NY 14263 (E-mail: chumy.nwogu@ roswellpark.org).

J Thorac Cardiovasc Surg 2015;149:71-2

$0022-5223 / \$ 36.00$

Copyright (c) 2015 by The American Association for Thoracic Surgery http://dx.doi.org/10.1016/j.jtcvs.2014.09.127
}

Sato and colleagues ${ }^{2,3}$ from Japan have provided more evidence-based insight into lung resections in patients with ILD using a much larger cohort of patients from 61 different institutions. They retrospectively analyzed 1763 patients with non-small cell lung cancer who underwent pulmonary resection with a clinical diagnosis of ILD over a 10-year period. They reported an incidence of operation-induced acute exacerbation (AE) of ILD in this patient group of $9.3 \%$ and a mortality rate of $43.9 \%{ }^{2}$ The lobectomy/segmentectomy and the bilobectomy/pneumonectomy groups were both more likely to develop $\mathrm{AE}$ than the wedge resection group with odd ratios of 3.83 and 5.70, respectively. However, the 5-year survival of patients with stage IA disease was $33.2 \%, 61.0 \%$, and $68.4 \%$ for the wedge resection, segmentectomy, and lobectomy groups, respectively $(P=.0038){ }^{3}$ The leading cause of death was cancer recurrence $(50.2 \%)$ followed by respiratory failure $(26.8 \%)$. The 5-year survival of the patients with stage IA disease with percent vital capacity $(\% \mathrm{VC})$ of $80 \%$ or less was $20 \%$, whereas those with $\% \mathrm{VC}$ greater than $80 \%$ was $64.3 \%$ (log-rank test, $P<.0001)$. Although DLCo is a well-known survival predictor for patients with ILD, they did not include it in their analysis because of too many missing values in their cohort of patients. Performing the procedure by video-assisted thoracoscopic surgery did not reduce the risk of acute exacerbation of ILD. The size of the patient cohort, multi-institutional participation and long follow-up are strengths of the study. However, its weaknesses include the lack of clear selection criteria for their patients, the absence of DLco from the predictive analysis, and the use of predicted $\% \mathrm{VC}$ without consideration of the extent of the lung resection. It would be enlightening to assess whether calculation of predicted postoperative FVC based on the required lung volume required for an R0 resection, would predict outcomes for segmentectomies, lobectomies, and even pneumonectomies.

It has been suggested that certain intraoperative maneuvers may improve outcomes in patients with ILD. These include shortening the duration of one-lung ventilation, avoiding fluid overload or high inspired oxygen concentration, limiting the extent of resection (ie, avoiding pneumonectomy), and the use of sublobar resection such as segmentectomy when anatomically and oncologically appropriate. ${ }^{1}$

In conclusion, major lung resection in patients with ILD is associated with increased postoperative morbidity and mortality. Patients with a low preoperative FVC should be carefully assessed before undergoing surgery, particularly 
in the presence of a lower DLco. ${ }^{1}$ Pneumonectomy carries a prohibitive surgical risk in patients with ILD. However, long-term survival is still achievable in a substantial subgroup of these patients undergoing lesser surgical procedures. Wedge resections are better tolerated but result in less long-term survival. Thus, anatomic surgical resections can be offered to appropriately selected patients with lung cancer and ILD, keeping in mind the risk of respiratory failure during the evaluation of such patients. Although late outcomes after lung resection are uniformly worse in patients with ILD compared with patients without ILD, long-term survival is still achievable in a superselected group. ${ }^{1}$ The long-term results presented by Sato and col- leagues further equip thoracic surgeons to properly select suitable surgical candidates.

\section{References}

1. Voltolini L, Bongiolatti S, Luzzi L, Bargagli E, Fossi A, Ghiribelli C, et al. Impact of interstitial lung disease on short-term and long-term survival of patients undergoing surgery for non-small-cell lung cancer: analysis of risk factors. Eur J Cardiothorac Surg. 2013;43:e17-23.

2. Sato T, Teramukai S, Kondo H, Sato T, Watanabe A, Ebina M, et al. Impact and predictors of acute exacerbation of interstitial lung diseases after pulmonary resection for lung cancer. J Thorac Cardiovasc Surg. 2014;147:1604-11.

3. Sato T, Kondo H, Teramukai S, Kishi K, Ebina M, Sugiyama Y, et al. Longterm results and predictors of survival after surgical resection of the lung cancer patients with interstitial lung diseases. J Thorac Cardiovasc Surg. 2015; 149:64-70. 